\title{
The Influence of Machine Saturation on Bifurcation and Chaos in Multimachine Power Systems
}

\author{
Majdi M. Alomari and Jian Gue Zhu
}

University of Technology, Sydney (UTS)

P.O. Box 123, Broadway NSW 2007, Australia

Email: majdi.m.alomari@eng.uts.edu.au

\begin{abstract}
A bifurcation theory is applied to the multimachine power system to investigate the effect of iron saturation on the complex dynamics of the system. The second system of the IEEE second benchmark model of Subsynchronous Resonance (SSR) is considered. The system studied can be mathematically modeled as a set of first order nonlinear ordinary differential equations with $\left(\mu=X_{c} / X_{L}\right)$ as a bifurcation parameter. Hence, bifurcation theory can be applied to nonlinear dynamical systems, which can be written as $d x / d t=F(x ; \mu)$. The results show that the influence of machine saturation expands the unstable region when the system loses stability at the Hopf bifurcation point at a less value of compensation.
\end{abstract}

Keywords: Machine Saturation, Hopf Bifurcation, Chaos, Subsynchronous Resonance, Damper Windings.

\section{Introduction:}

Addition of series compensation in power systems, which is considered for increasing effectively the power transfer capability as well as improving the stability, introduces a problem called subsynchronous resonance (SSR). Subsynchronous resonance can be defined as electromechanical interaction between electrical resonant circuits of the transmission system and the torsional natural frequencies of the turbinegenerator rotor. Where the torques produced by the interaction of the subharmonic current in the stator RLC circuit and the rotor circuits of the synchronous generator may excite one of the natural torsional modes of oscillation of the turbine-generator masses leading to shaft failure. Three types of SSR can identify the interaction of the system and the generator under the subsynchronous resonance. They have been called torsional interaction effect, induction generator effect, and transient torque effect. In this research, we focus on the torsional interaction effect, which results from the interaction of the electrical subsynchronous mode with the torsional mode. On the other hand, several methods have been used in SSR study. The most common of these methods are eigenvalue analysis, frequency scanning, and timedomain analysis. The eigenvalue analysis is used in this study. It is a very valuable technique because it provides both the frequencies of oscillation and the damping at each frequency.

Abed and Varaiya [1] employed Hopf bifurcation theory to explain nonlinear oscillatory behavior in power systems. The bifurcation theorem was used by Zhu et al [2] to demonstrate the existence of a Hopf bifurcation in a single machine infinite busbar (SMIB) power system, in which the dynamics of the damper windings and the AVR are neglected. Tomim et al [3] proposed an index that identifies Hopf bifurcation points in power systems susceptible to subsynchronous resonance. Nayfeh et al [4] applied the bifurcation theory to a practical series capacitor compensated single machine power system, the BOARDMAN turbine-generator system. To make the analysis simple and manageable, the effect of stator and rotor iron saturation in synchronous machines was neglected in all of the bifurcation analysis given in the literature. However, the importance of saturation has been recognized by many authors [5, 6, 7]. The effect of electrical machine saturation on SSR was studied by Harb et al [8]. They concluded that the generator saturation slightly shrinks the positively damped region by shifting the Hopf bifurcation point, secondary Hopf bifurcation and bluesky catastrophe to smaller compensation level. 
In this research, power system dynamics has been studied using the nonlinear dynamics point of view, which utilizes the bifurcation theory. Bifurcation theory and chaos is used to investigate the complex dynamics of the considered system. The system loses stability via Hopf bifurcation point $(H)$ and a limit cycle is born at $\mu=H$. This limit cycle is stable if the bifurcation is supercritical and unstable if it is subcritical. The type of the Hopf bifurcation is determined by numerical integration of the system, with specific amount of initial disturbances, slightly before and after the bifurcation value. On further increase of the compensation factor, the system experiences chaos via torus attractor. Chaos is a bounded steadystate behavior that is not an equilibrium solution or a periodic solution or a quasiperiodic solution [9]. In this paper, we study the effect of iron saturation on Bifurcation and Chaos.

\section{System Description}

The system considered is the two different machine infinite bus system, shown in Figure 1. The two machines have a common torsional mode connected to a single series compensated transmission line. The model and the parameters are provided in the second system of the IEEE second benchmark model. The electro-mechanical systems for the first and second units are shown in Figure. 2. The first unit consists of exciter (EX.), generator (Gen.1), low-pressure (LP1) and high-pressure (HP1) turbine sections. And the second unit consists of generator (Gen.2), low-pressure (LP2) and high-pressure (HP2) turbine sections. Every section has its own angular momentum constant $\mathrm{M}$ and damping coefficient $\mathrm{D}$, and every pair of successive masses have their own shaft stiffness constant K, as shown in Figure 2. The data for electrical and mechanical system are provided in [10]. Replacement of these generators with a single equivalent generator will change the resonance characteristics and therefore is not justified. Consequently, each generator is represented in its own rotor frame of reference and suitable transformation is made.

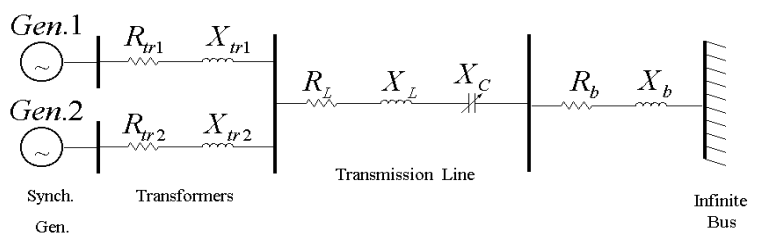

Fig. 1. Electrical system ( Two different machine infinite bus system ).

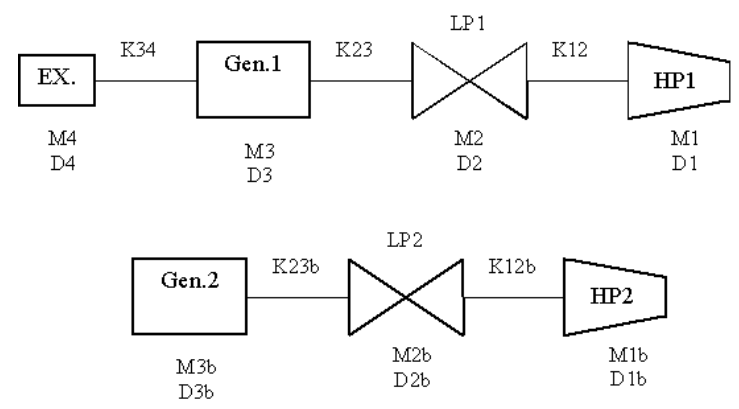

Fig. 2. Electro-mechanical systems for the first and second units.

\section{Mathematical Model}

The mathematical model of the electrical and mechanical system will be presented in this section. Actually, the electrical system includes the dynamic nonlinear mathematical model of a synchronous generator and that of the transmission line. The generator model considered in this study includes five equations, d-axis stator winding, q-axis stator winding, d-axis rotor field winding, q-axis rotor damper winding and d-axis rotor damper winding equations. Each mass of the mechanical system can be modeled by a second order ordinary differential equation (swing equation), which is presented in state space model as two first order ordinary differential equations. Using the direct and quadrature d-q axes and Park's transformation, we can write the complete mathematical model that describes the dynamics of the system. The mathematical model of the electrical and mechanical system is provided in [11]. 


\section{System Response in Case of absence of Saturation}

In this section, we study the case of adding damper winding on subsynchronous resonance of the first generator but neglecting saturation. In case of no saturation, we set $d_{0}, d_{1}, d_{2}, d_{3}, q_{0}, q_{1}, q_{2}$, and $q_{3}$ equal to zero in equations (1) and (2). In this case we have 23 ordinary nonlinear differential equations. The equilibrium solution is obtained by setting the derivatives of the 23 state variables in the system equal to zero. The stability of the operating point is studied by examination of the eigenvalues of the linearized model evaluated at the operating point. The operating point stability regions in the $\delta_{r 1}-\mu$ plane together with two Hopf bifurcation points are depicted in Figure 3.
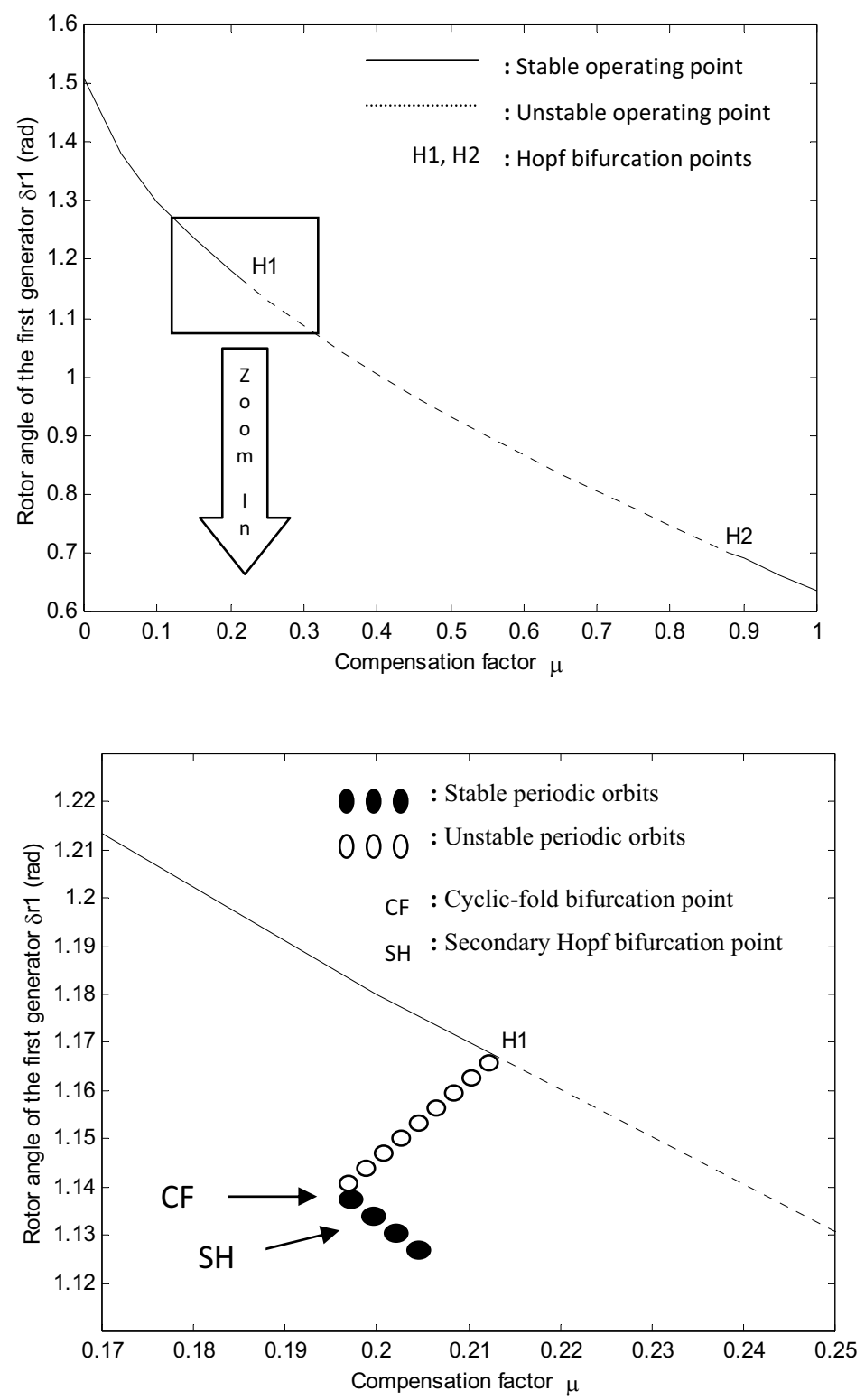

Fig. 3 Bifurcation diagram showing variation of the first generator rotor angle $\delta_{r 1}$ with the compensation factor $\mu$ (for the case of absence of Saturation). 
We observe that the power system has a stable operating point to the left of $H_{1} \approx 0.212486$ and to the right of $H_{2} \approx 0.880735$, and has an unstable operating point between $H_{1}$ and $H_{2}$. The operating point loses stability at a Hopf bifurcation point, namely $\mu=H_{1}$. It regains stability at a reverse Hopf bifurcation, namely $\mu=\mathrm{H}_{2}$. In this case a pair of complex conjugate eigenvalues will transversally cross from left half to right half of the complex plane, and then back to the left half.

To determine whether the limit cycles created due to the Hopf bifurcation are stable or unstable, we obtain the time response of the system by numerical integration with small disturbance slightly before $H_{1}$. Figure 4 shows the response of the system with 7\% initial disturbance on the speed of the generator at $\mu=0.199135$, which is less than $H_{1}$. It can be observed that the system is unstable. Therefore, the type of this Hopf bifurcation is subcritical. So, the periodic solution emanating at the bifurcation point is unstable.
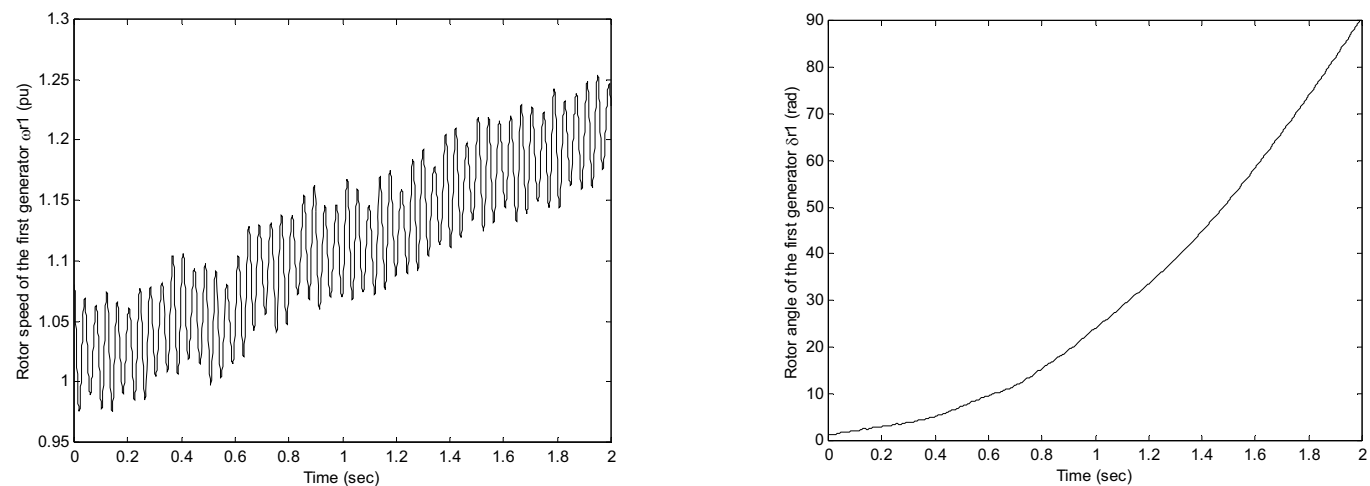

Fig. 4 Response of the system. Rotor speed of the generator (left) and rotor angle of the generator (right) at $\mu=0.199135$ with $7 \%$ initial disturbance in rotor speed of generator (for the case of absence of Saturation).

Locally, the system has a stable equilibrium solution and a small unstable limit cycle for values of $\mu$ slightly before $H_{1}$. As $\mu$ increases past $H_{1}$, the equilibrium solution loses stability and the small unstable limit cycle disappears. As $\mu$ decreases below $H_{1}$, the unstable limit cycle deforms and increases in size until the branch of unstable limit cycles meets a branch of stable large-amplitude limit cycles at $\mu=0.198526$, corresponding to point $C F$. As the value of compensation factors decreases more, the stable and unstable limit cycles collide and exterminate each other in a cyclic-fold bifurcation. As $\mu$ increases from $C F$, the stable limit cycle grows in size while remaining stable. The limit cycle undergoes a secondary Hopf bifurcation at $\mu=S H \approx 0.201325$, resulting in a two-period quasiperiodic attractor. While it oscillates with more than one incommensurate frequency slightly after the secondary Hopf bifurcation point at $\mu=0.201350$ and obtain a period quasiperiodic (torus) attractor. As $\mu$ increases slightly after $S H$ up to $\mu=0.206433$, the chaotic attractor is obtained. Figure 5 shows the two-dimensional projections and time histories of the system at different compensation factors. 
(a)

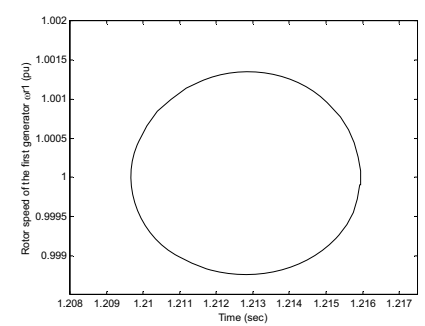

(b)

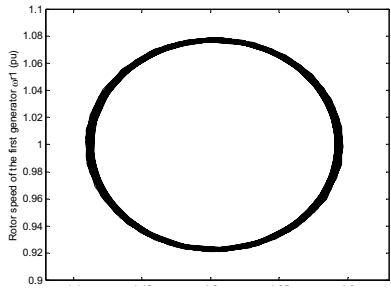

(c)

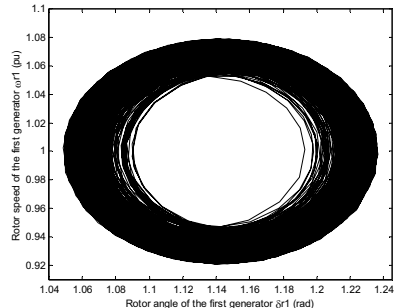

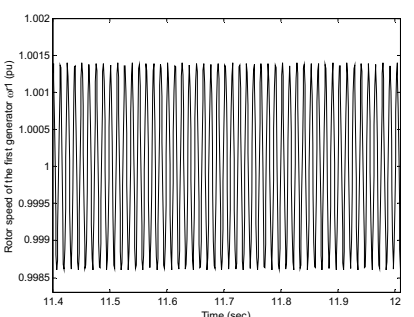
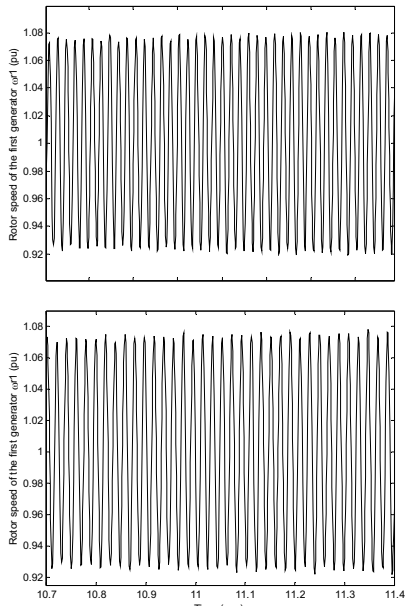

Fig. 5 Two-dimensional projection of the phases portrait onto $\omega_{r n}-\delta_{r n}$ plane (left) and the time histories of the corresponding rotor speed of generator (right). The solution at (a) limit cycle, $\mu=0.198526$, (b) torus-attractor, $\mu=0.201350$ and (c) chaotic attractor, $\mu=0.206433$ (for the case of absence of Saturation).

\section{System Response in Presence of Saturation}

In this section, we investigate the influence of iron saturation in both the $\mathrm{d}$ - and q-axes on the subsynchronous instability of the first generator. This is done by performing a bifurcation analysis in a complete model of the system. The model includes saturation but neglects the dynamics of the automatic voltage regulator (AVR) and the turbine governor. When researching iron saturation for stability studies, it is assumed that the saturation relationship between the resulting air-gap flux and the mmf under loaded conditions is the same as in no-load conditions. This allows the saturation characteristics to be represented by the open-circuit saturation curve.

When saturation is taken into consideration in both the d- and q-axes, it leads to an improvement in the accuracy of the simulation models. When performing iron saturation in stability studies, the following assumptions are made [5]. First, the leakage fluxes are in air for a large portion of their paths. Therefore, they are minimally affected by saturation of the iron core. So, the leakage inductances are independent of the iron saturation. Consequently mutual flux linkages $\Psi_{m d}$ and $\Psi_{m q}$ are the only elements that saturate are the mutual flux linkages. Second, the leakage fluxes are usually small and their paths coincide with that of the main flux for only a small part of its path. So, saturation can be determined by the air-gap flux linkage only. Third and final assumption is that the saturation relationship between the resultant air-gap flux and 
the mmf under loaded conditions is the same as in no-load conditions. This allows the saturation characteristics to be represented by the open-circuit saturation curve.

In order to obtain the generator analytical formulas for the fluxes of the first generator $\Psi_{m d l}$ and $\Psi_{m q 1}$ as functions of the currents, Harb et al [8] fitted the test data which were discussed by Minnich et al [12]. The result was the following third-order polynomial functions:

$$
\begin{aligned}
& \psi_{m d 1}=d_{0}+d_{1}\left(i_{f 1}-i_{d 1}\right)+d_{2}\left(i_{f 1}-i_{d 1}\right)^{2}+d_{3}\left(i_{f 1}-i_{d 1}\right)^{3} \\
& \psi_{m q 1}=q_{0}+q_{1}\left(i_{Q 1}-i_{q 1}\right)+q_{2}\left(i_{Q 1}-i_{q 1}\right)^{2}+q_{3}\left(i_{Q 1}-i_{q 1}\right)^{3}
\end{aligned}
$$

where $d_{0}, d_{1}, d_{2}, d_{3}, q_{0}, q_{1}, q_{2}$ and $q_{3}$ are constants. Clearly, the mutual flux linkages of the first generator $\Psi_{m d l}$ and $\Psi_{m q 1}$ are nonlinear functions of the first machine currents $i_{d 1}, i_{q 1}, i_{f 1}$, and $i_{Q 1}$. The flux linkages of the first generator $\Psi_{d l}$ and $\Psi_{q l}$ of the d- and q-axes can be determined from the mutual flux linkages of the first generator $\Psi_{m d l}$ and $\Psi_{m q l}$ as follows:

$$
\begin{aligned}
& \psi_{d 1}=\psi_{m d 1}-X_{l e} i_{d 1} \\
& \psi_{q 1}=\psi_{m q 1}-X_{l e} i_{q 1} \\
& \psi_{f 1}=\psi_{m d 1}-X_{l e} i_{f 1} \\
& \psi_{Q 1}=\psi_{m q 1}-X_{l e} i_{Q 1}
\end{aligned}
$$

where $X_{l e}$ is the leakage reactance.

In this formulation, the dynamics of the automatic voltage regular (AVR) and the turbine governor are neglected. The dynamics of the damper windings as well as the machine saturation on the both axes of the first generator are included. We will show that machine saturation reduces the compensation level at which subsynchronous resonance occurs.

The operating point stability regions in the $\delta_{r 1}-\mu$ plane together with two Hopf bifurcation points are depicted in Figure 6. We observe that the power system has a stable operating point to the left of $H_{1} \approx 0.174653$ and to the right of $H_{2} \approx 0.856765$, and has an unstable operating point between $H_{1}$ and $H_{2}$. The operating point loses stability at a Hopf bifurcation point where $\mu=H_{1}$. It regains stability at a reverse Hopf bifurcation for $\mu=H_{2}$. In this case a pair of complex conjugate eigenvalues will transversally cross from left half and right half of the complex plane, and then back to left half. The results show that machine saturation reduces the compensation level at which subsynchronous resonance occurs. The generator saturation slightly shrinks the positively damped region by shifting the Hopf bifurcation point to smaller compensation level.

\section{Conclusions}

We have applied bifurcation theory to investigate the influence of iron saturation. The case of neglecting the saturation is considered. The results showed that as the compensation factor increases the operating point loses stability via subcritical Hopf bifurcation point. When the machine saturation is considered, the results showed that the generator saturation shifts the subcritical Hopf bifurcation to smaller value of $\mu$ and hence shrinks the stable region. In other words, the generator saturation destabilize the system by reducing the compensation level at which subsynchronous resonance occurs. It also slightly shifts the secondary Hopf bifurcation and bluesky catastrophe to smaller compensation level. 


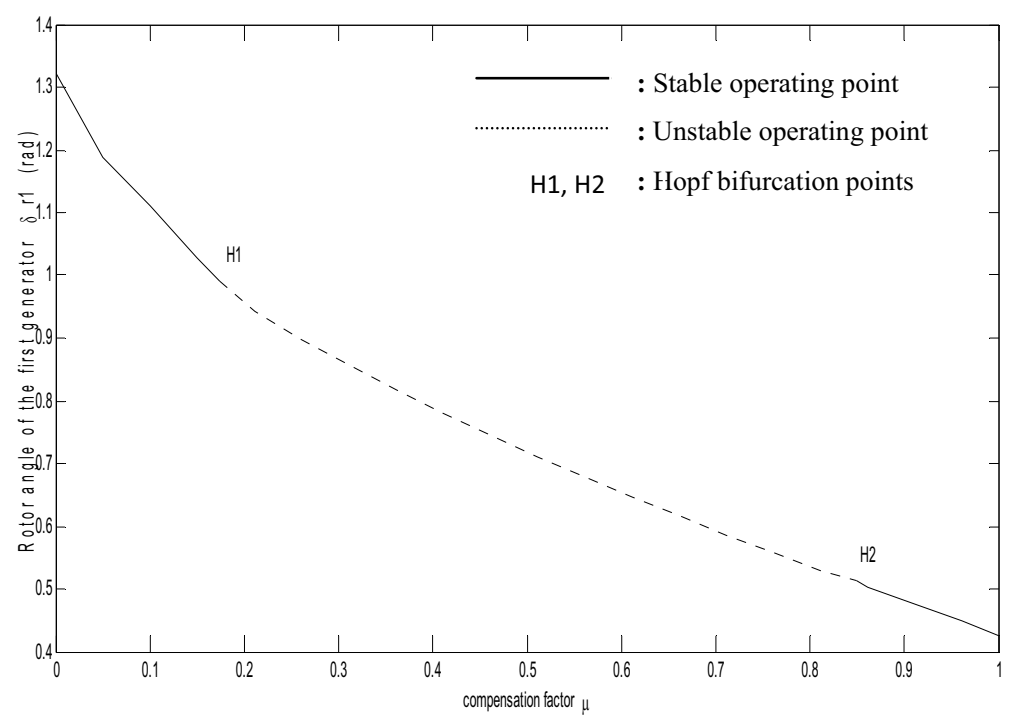

Fig. 6 Bifurcation diagram showing variation of the first generator rotor angle $\delta_{r 1}$ with the compensation factor $\mu$ (for the case of adding saturation to the first generator).

\section{References}

[1] E. H. Abed and P. P. Varaiya, "Nonlinear Oscillations in Power Systems", Int. J. Elecr. Power and Energy System, Vol. 6, pp. 37-43, Jan., 1984.

[2] W. Zhu, R.R. Mohler, R. Spce, W.A. Mittelstadt and D. Maratukulman, "Hopf Bifurcation in a SMIB Power System with SSR,” IEEE Trans. on Power Systems, Vol.11, No.3, pp. 1579-1584, 1996.

[3] M. A. Tomim, A. C. Zambroni de Souza, P. P. Carvalho Mendes, and G. Lambert-Torres, "Identification of Hopf Bifurcation in Power Systems Susceptible to Subsynchronous Resonance", IEEE Bologna Power Tech Conference, June 23-26, 2003, Bologna, Italy.

[4] A.H Nayfeh, A.M. Harb, and C-M.Chin, A.M. Hamdan and L. Mili, Application of Bifurcation Theory to Subsynchronous Resonance in Power Systems, Int.J.Bifurcation and Chaos,Vol.8,No.1,pp.157-172, 1998

[5] Kundur, P. 1994, Power System Stability and Control, Electric Power Research Institute, McGrawHill, New York.

[6] Harly, R. G., Limebeer, D. J., and Chirricozzi, E. , 1980, "Comparative study of the saturation methods in synchronous machine models," IEEE Proceedings, Vol. 127, Part B.

[7] De Mello, F. P., and Hannet, L. N. "Representation of Saturation in Synchronous Machines," IEEE Transactions on Power Systems PWRS-1, 1986.

[8] A.M. Harb, L. Mili, A.H Nayfeh and C-M. Chin, On the Effect of the Machine Saturation on SSR in Power Systems, Electrical Machines and Power Systems, Vol.20, pp. 1019-1035, 2000.

[9] A.H. Nayfeh and B. Balachandran, Applied Nonlinear Dynamics, Wiley, New York, 1995. 
Majdi M. Alomari and Jian Gue Zhu

[10] IEEE SSR Working Group, "Second Benchmark Model for Computer Simulation of Subsynchronous Resonance,” IEEE Trans. On Power Apparatus and Systems. Vol. PAS- 104, No.5, 1057-1064, May 1985.

[11] Majdi M. Alomari and Benedykt S. Rodanski, "The Effects of Machine Components on Bifurcation and Chaos as Applied to Multimachine System”, CHAOS2008, Chaotic Modeling and Simulation International Conference, Chania, Crete, Greece, 3-6 June 2008.

[12] S. H. Minnich, R. P. Schulz, D. H. Baker, D. K. Sharma, R. G. Farmer, and J. H. Fish, "Saturation Functions for Synchronous Generators from Finite Elements", IEEE Trans. Energy Conversion, vol. EC-2, December 1987. 